\title{
Imaging and controlling electron transport inside a quantum ring
}

\author{
B. HACKENS ${ }^{1,2 *}$, F. MARTINS ${ }^{1}$, T. OUISSE ${ }^{1}$, H. SELLIER ${ }^{1}$, S. BOLLAERT ${ }^{3}$, X. WALLART ${ }^{3}$, A. CAPPY ${ }^{3}$, \\ J. CHEVRIER ${ }^{4}$, V. BAYOT ${ }^{2}$ AND S. HUANT1* \\ ${ }^{1}$ Laboratoire de Spectrométrie Physique, Université Joseph Fourier Grenoble and CNRS, 140 rue de la Physique, 38402 Saint Martin d'Hères, France \\ ${ }^{2}$ CERMIN, DICE Lab, Université Catholique de Louvain, 3 Place du Levant, 1348 Louvain-la-Neuve, Belgium \\ ${ }^{3}$ IEMN, Avenue Poincarè, BP 60069, 59652 Villeneuve d'Ascq, France \\ ${ }^{4}$ LEPES, CNRS, 25 avenue des Martyrs, 38042 Grenoble, France \\ *e-mail: hackens@dice.ucl.ac.be; serge.huant@ujf-grenoble.fr
}

T raditionally, the understanding of quantum transport, coherent and ballistic ${ }^{1}$, relies on the measurement of macroscopic properties such as the conductance. Although powerful when coupled to statistical theories, this approach cannot provide a detailed image of 'how electrons behave down there'. Ideally, understanding transport at the nanoscale would require tracking each electron inside the nanodevice. Significant progress towards this goal was obtained by combining scanning probe microscopy with transport measurements ${ }^{2-7}$. Some studies even showed signatures of quantum transport in the surroundings of nanostructures ${ }^{4-6}$. Here, scanning probe microscopy is used to probe electron propagation inside an open quantum ring exhibiting the archetype of electronwave interference phenomena: the Aharonov-Bohm effect ${ }^{8}$. Conductance maps recorded while scanning the biased tip of a cryogenic atomic force microscope above the quantum ring show that the propagation of electrons, both coherent and ballistic, can be investigated in situ, and can even be controlled by tuning the potential felt by electrons at the nanoscale.

An open quantum ring $(\mathrm{QR})$ in the coherent regime of transport is a good example of an interferometer: its conductance peaks when electron waves interfere constructively at the output contact and decreases to a minimum for destructive interferences. Varying either the magnetic flux encircled by the QR or the electrostatic potential in one arm allows the interference to be tuned. This gives rise to the well-known magnetic ${ }^{9}$ and electrostatic ${ }^{10,11}$ Aharonov-Bohm (AB) oscillations. Although these effects have been studied extensively through transport measurements, those techniques lack the spatial resolution necessary to probe interferences in the interior of QRs. In this work, we perturb the propagation of electrons through a QR with an atomic force microscope (AFM) tip. We therefore take advantage of both the imaging capabilities of the AFM and the high sensitivity of the conductance measurement to electron phase changes.
A three-dimensional image of the $\mathrm{QR}$ used here, as measured by our AFM in the conventional topographical mode, is shown in Fig. 1a. The QR is fabricated from an InGaAs/InAlAs heterostructure hosting a two-dimensional electron system (2DES) with a sheet density of $2 \times 10^{16} \mathrm{~m}^{-2}$, buried $25 \mathrm{~nm}$ below the sample surface ${ }^{12}$. Electron-beam lithography and wet etching were used to pattern the QR and interconnections. At the experimental temperature $(4.2 \mathrm{~K})$, the $\mathrm{QR}$ is smaller than the intrinsic electron mean free path measured in the $2 \mathrm{DES}\left(l_{\mu}=2.3 \mu \mathrm{m}\right)$. Transport is thus in the ballistic regime, with electrons travelling along 'billiardball'-like trajectories. Moreover, the observation of periodic AB oscillations (Fig. 1b, inset) in the magnetoconductance of our QR attests that transport is also in the coherent regime ${ }^{13}$. The periodicity of these oscillations is found to be $26 \mathrm{mT}$, consistent with the average radius of circular electron trajectories in the QR: $r=220 \mathrm{~nm}$.

The metallized tip of the AFM is biased at a voltage $V_{\text {tip }}=0.3 \mathrm{~V}$ and scanned in a plane parallel to the 2DES, at a typical tip-2DES distance of $50 \mathrm{~nm}$. The tip acts as a flying nanogate that modifies locally the electrostatic potential experienced by electrons within the QR. The transmission of electrons through the QR is then affected by the action of this perturbating potential thanks to two distinct effects: (1) the modification of electron wavelength and phase in the perturbed area (coherent effect), and (2) the change in momentum of electrons impinging the local perturbation (semiclassical effect). The conductance of the sample, which is a measure of electron transmission, is recorded as the tip scans over the QR to provide a two-dimensional conductance map (Fig. 1c).

The first-order contribution to the conductance map shown in Fig. 1c is a broad background structure extending beyond the limits of the QR (note that a similar background was observed in the case of large open quantum dots ${ }^{7}$ ). Its overall shape is strongly affected by successive illuminations of the sample, which are known to affect the configuration of ionized impurities. On the other 

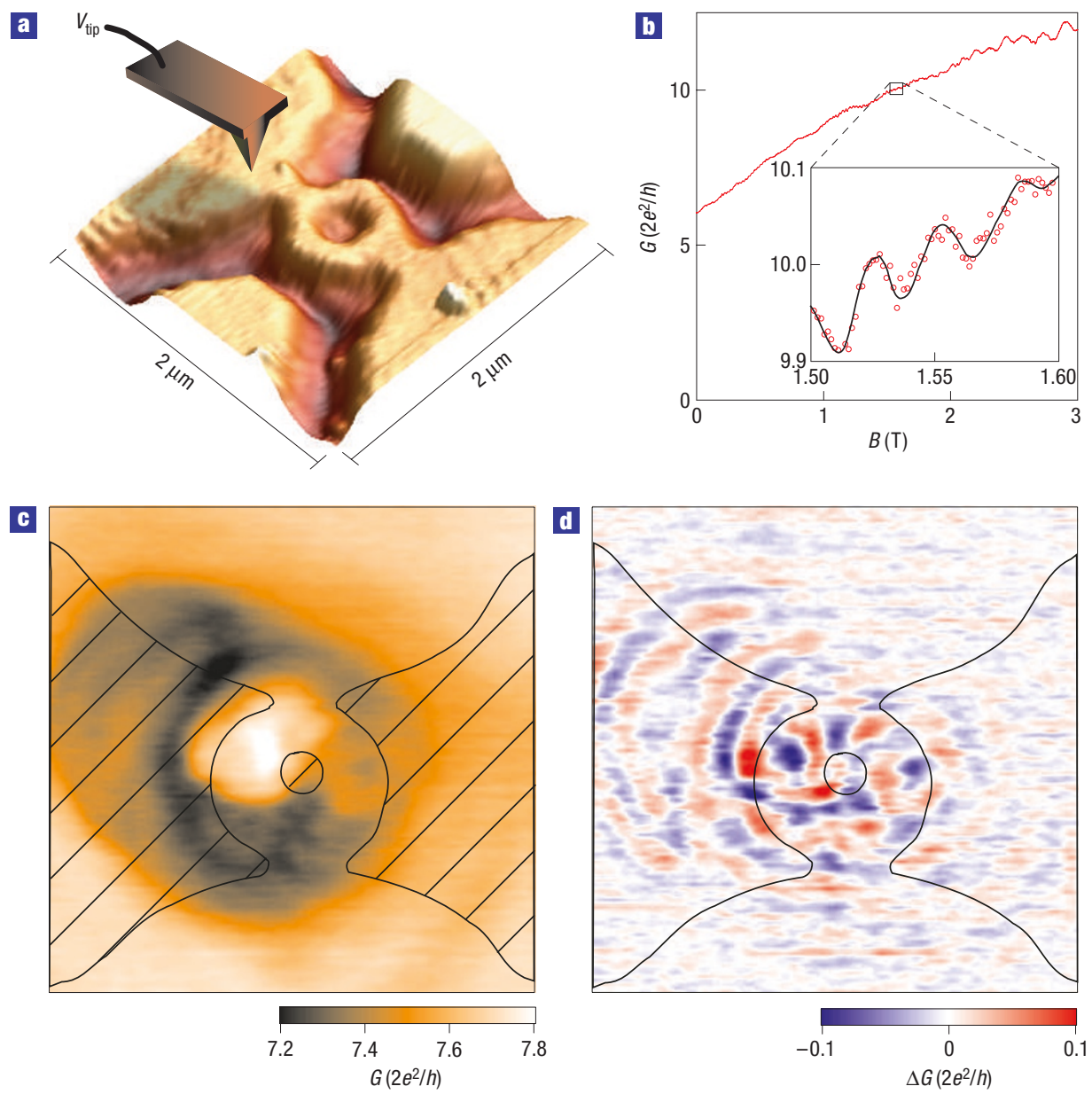

Figure 1 Experimental setup and conductance maps. a, Three-dimensional reconstruction of the QR topography, on the basis of an AFM image. The inner and outer ring diameters are 210 and $600 \mathrm{~nm}$, respectively. Conductance maps are obtained by biasing the AFM tip and scanning it at a distance from the 2DES, as schematically represented, and simultaneously measuring the conductance of the $\mathrm{QR}$. b. Conductance of the QR as a function of the magnetic field, measured at $4.2 \mathrm{~K}$ using a lock-in technique, with a probe current of $20 \mathrm{nA}$ and a frequency of $1.574 \mathrm{kHz}$. From the average conductance level, we deduce that the number of quantum modes in the openings of the $\mathrm{QR}$ is larger than 6 . The inset shows the magnetoconductance over a small range of $B$, exhibiting the periodic $h / e \mathrm{AB}$ oscillations. Above $B=2.2 \mathrm{~T}$, as the cyclotron radius shrinks below the width of the QR arms and openings, electrons move along the edges of the device and Shubnikov-de Haas oscillations develop. $\mathbf{c}$, Conductance of the QR as a function of the tip position, with $V_{\text {tip }}=0.3 \mathrm{~V}$, tip-2DES distance $d_{\text {tip }}=50 \mathrm{~nm}$ and $B=2 \mathrm{~T}$. A strong long-range background is clearly visible, with smaller range fringes superimposed. The position of the $\mathrm{QR}$, recorded in AFM topography mode before the conductance imaging, is indicated as a plain line. $\mathbf{d}$, High-pass-filtered conductance map, exhibiting small-range fringes.

hand, this background remains insensitive to the magnetic field $B$. This indicates that the background is not related to quantum transport, neither coherent nor ballistic ${ }^{12}$, but rather to a global shift of the electric potential in the whole QR as the tip approaches the device.

A closer look at the conductance map reveals that the broad background is decorated by a more complex pattern of smaller scale features, particularly visible in the central part of the image. We will see that this second-order effect shares a common feature with quantum transport inside the QR: its dependence on magnetic field. A high-pass filter applied to the raw conductance map reveals clear conductance oscillations (Fig. 1d) whose physical origin will be investigated in the remainder of the paper. The typical spatial periodicity of the oscillations $(\sim 100 \mathrm{~nm})$ is much larger than the electron Fermi wavelength in our sample $\left(\lambda_{\mathrm{F}} \sim 20 \mathrm{~nm}\right)$. This tells us immediately that the 'standing electron wave' pattern observed in previous experimental studies ${ }^{5,6}$ is not the relevant mechanism to explain our observations. It is worth noting that the amplitude of filtered conductance $(\Delta G)$ fringes in Fig. 1d is larger on the left side of the QR than on its right side, in contrast with the usual assumption of a symmetric role of the two arms in $\mathrm{AB}$ rings. This left-right imbalance is observed whatever the direction of the magnetic field or the probe current. Therefore, it is related to an asymmetry of the QR or tip shape, and is not a signature of the Lorentz force, which could also lead to an imbalance of the electron injection in the two arms of the QR. Indeed, such an asymmetry is not surprising, as it has already been shown in otherwise lithographically symmetric side-gated $\mathrm{QR}^{11}$.

Most interestingly, the data in Fig. 1d reveal that the type of fringe pattern depends on the scanned region. Whereas fringes are predominantly radial when the tip is located directly above the ring, they become concentric when the tip moves away from the QR. Figure 2 highlights the fundamental difference between both types of fringes as shown by the influence of an added bias current 

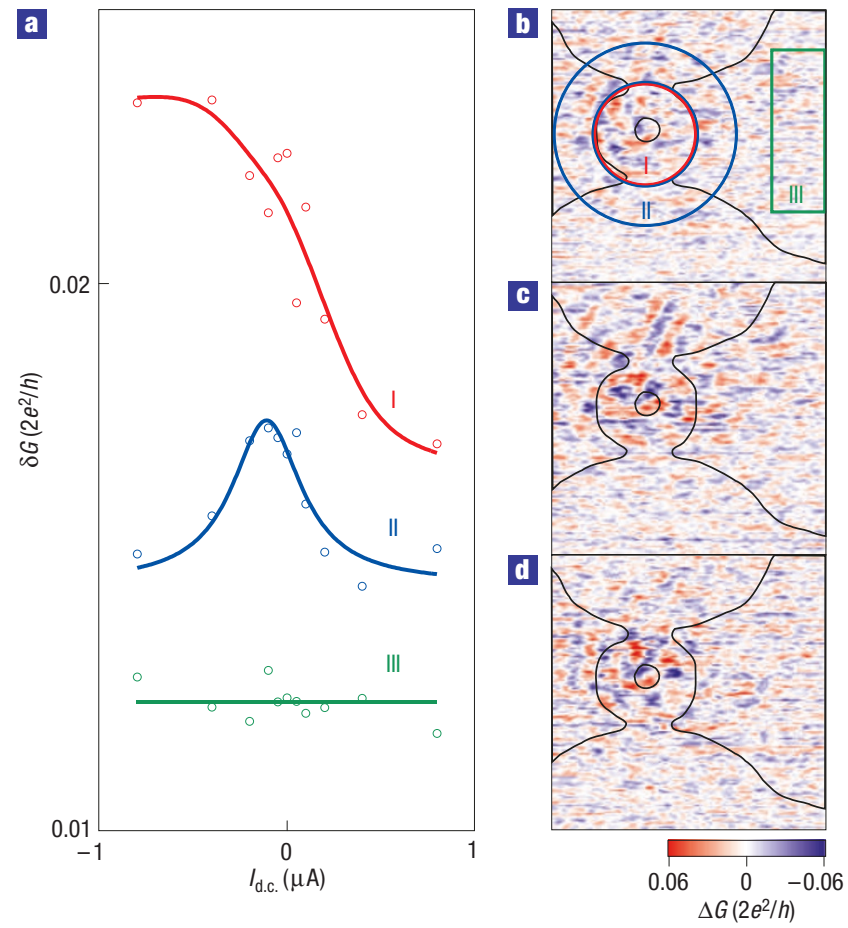

Figure 2 Evidence for a different behaviour of the standard deviation of radial and concentric fringes. a, $\delta G$ (standard deviation of $\Delta G$ ) as a function of $I_{\text {d.c. }}$, calculated in different areas (I-III) of the conductance maps, indicated in $\mathbf{b}$. The asymmetric evolution in region I is a signature of ballistic effects, influenced by the sign of the probe current, whereas the symmetric decay in region II is related to the coherent nature of electron waves. The lines are guides to the eye.

b-d, Conductance maps recorded at $B=5.95 \mathrm{~T}$ for different bias currents $I_{\text {d.c. }}:+0.8 \mu \mathrm{A}(\mathbf{b}), 0 \mu \mathrm{A}(\mathbf{c})$ and $-0.8 \mu \mathrm{A}(\mathbf{d})$.

$I_{\text {d.c. }}$ on the conductance map. Increasing the absolute value of $I_{\text {d.c. }}$ is equivalent to raising the electron excess energy relative to the Fermi energy ${ }^{14}$. The bias current applied in Fig. 2b,d brings the electron system out of thermal equilibrium by more than $1 \mathrm{meV}$. We analyse the data by dividing the scan into three regions: the area enclosed in the QR (region I), a ring-shaped area in the vicinity of the QR (region II) and finally an area situated far from the QR where the influence of the tip vanishes (region III). First, we observe a strong reduction of concentric fringes (region II) at large bias irrespective of its sign. Region I, on the other hand, exhibits a very different behaviour depending on current direction: whereas conductance fringes die out at large positive bias (Fig. 2b), they strengthen and show a somewhat different pattern when bias is reversed (Fig. 2d).

A more quantitative picture of these differences is revealed in the evolution of $\delta G$, the standard deviation of $\Delta G$, calculated over regions I-III (Fig. 2a). Whereas region III exhibits no dependence on $I_{\text {d.c. }}$ and hence serves as a reference for the noise level, region II shows a fast decrease of $\delta G$ as $\left|I_{\text {d.c. }}\right|$ increases. The energy gained by electrons at high $\left|I_{\mathrm{d} . c .}\right|$ increases the electron-electron scattering rates ${ }^{14}$, which, in turn, suppresses phase-coherent effects. Therefore, this observation points towards a coherent origin for the concentric fringes in region II. In contrast, $\delta G$ in region I switches gradually from one level to another as $I_{\text {d.c. }}$ changes sign. As reversing the current produces a rearrangement of semiclassical ballistic trajectories inside the QR, the positive and negative current configurations induce different conductance maps (Fig. 2b-d).
Hence, the 'switching' behaviour observed in region I reflects the effect of the tip on semiclassical trajectories within the QR, and the symmetrical 'bell-shaped' behaviour in region II originates from the effect of the perturbating potential on the electron phase and wavelength.

The magnetic field, as it tunes the phase of interfering electrons, provides further valuable information on the origin of the fringes. Figure $3 \mathrm{a}-\mathrm{c}$ shows conductance maps measured at $B=1.5,1.513$ and $1.526 \mathrm{~T}$, covering a complete $\mathrm{AB}$ cycle, that is, the magnetic flux encircled in the area of the QR changes by one flux quantum $\left(\phi_{0}\right)$, implying that the dephasing of electron waves propagating through the two arms of the QR changes by $2 \pi$. As shown in the Supplementary Information video, concentric fringes expand continuously on increasing $B$. This is more clearly shown in Fig. $3 \mathrm{~d}$,e, which presents the evolution of average conductance profiles taken along two radii of the $\mathrm{QR}$ (regions $\alpha$ and $\beta$ in Fig. 3a) over an AB cycle. On both sides of the $\mathrm{QR}$, the oscillation pattern smoothly shifts away from the $\mathrm{QR}$ by one period as the $\mathrm{AB}$ phase $-\Delta \phi$-increases by one flux quantum. We interpret the behaviour observed in Fig. $3 \mathrm{~d}$,e as a scanning-gate-induced electrostatic $\mathrm{AB}$ effect. Indeed, as the tip approaches the $\mathrm{QR}$, either from the left or right, the electrical potential mainly increases on the corresponding side of the QR. This induces a phase difference between electron wavefunctions travelling through the two arms of the ring, which causes the observed oscillations. As the magnetic field applies an additional phase shift to electron wavefunctions, the V-shaped pattern formed by leftward- and rightward-moving fringes in Fig. 3d,e corresponds to isophase lines for the electrons. This observation ensures that our data are directly related to the $\mathrm{AB}$ effect and that concentric fringes originate from an interference effect of coherent electrons. Although the observed oscillations are reminiscent of those reported in QRs with biased side gates ${ }^{10,11}$, our experiment brings direct spatial information on interference effects, as we take advantage of the possibility to scan a localized perturbation across the sample.

We finally turn to the effect of magnetic field on the central part of our conductance maps. As shown in Fig. $3 \mathrm{a}-\mathrm{c}$ and in the Supplementary Information video, the evolution of the central pattern with $B$, although complex, always remains slow and smooth. This strongly contrasts with the much more unpredictable and faster evolution observed in experiments carried out on large open quantum dots ${ }^{7}$. We further note that the pattern of fringes observed at $B=2 \mathrm{~T}$ (Fig. 1d) is surprisingly similar to that visible at $B=1.5 \mathrm{~T}$ (Fig. $3 \mathrm{a}-\mathrm{c}$ ). This is a sign of the regular behaviour of the electron motion in $\mathrm{QR}$, in opposition to the chaotic evolution of the electron dynamics characterizing large open quantum dots. However, as the Fermi wavelength is much smaller than the width of the QR arms, a complete description of the evolution of the central pattern with $B$ would require in-depth simulation of the density of states inside the device taking into account the effect of the tip potential.

The present combination of AFM with transport measurements is very powerful for investigating electron interferences in real space and imaging ballistic transport at the local scale inside buried mesoscopic devices. It is predicted that this technique will also be useful in testing electronic devices based on realspace manipulation of electron interferences, such as the electronic analogues of optical or plasmonic components ${ }^{15}$ (resonators, Ysplitters and so on). In situ control over the electron potential would also provide a means to design new ballistic devices with desired characteristics (beam splitters, multi-terminal devices and so on). Therefore, our study paves the way for a wealth of experiments probing and controlling the local behaviour of charge carriers inside a large variety of open nanosystems. 

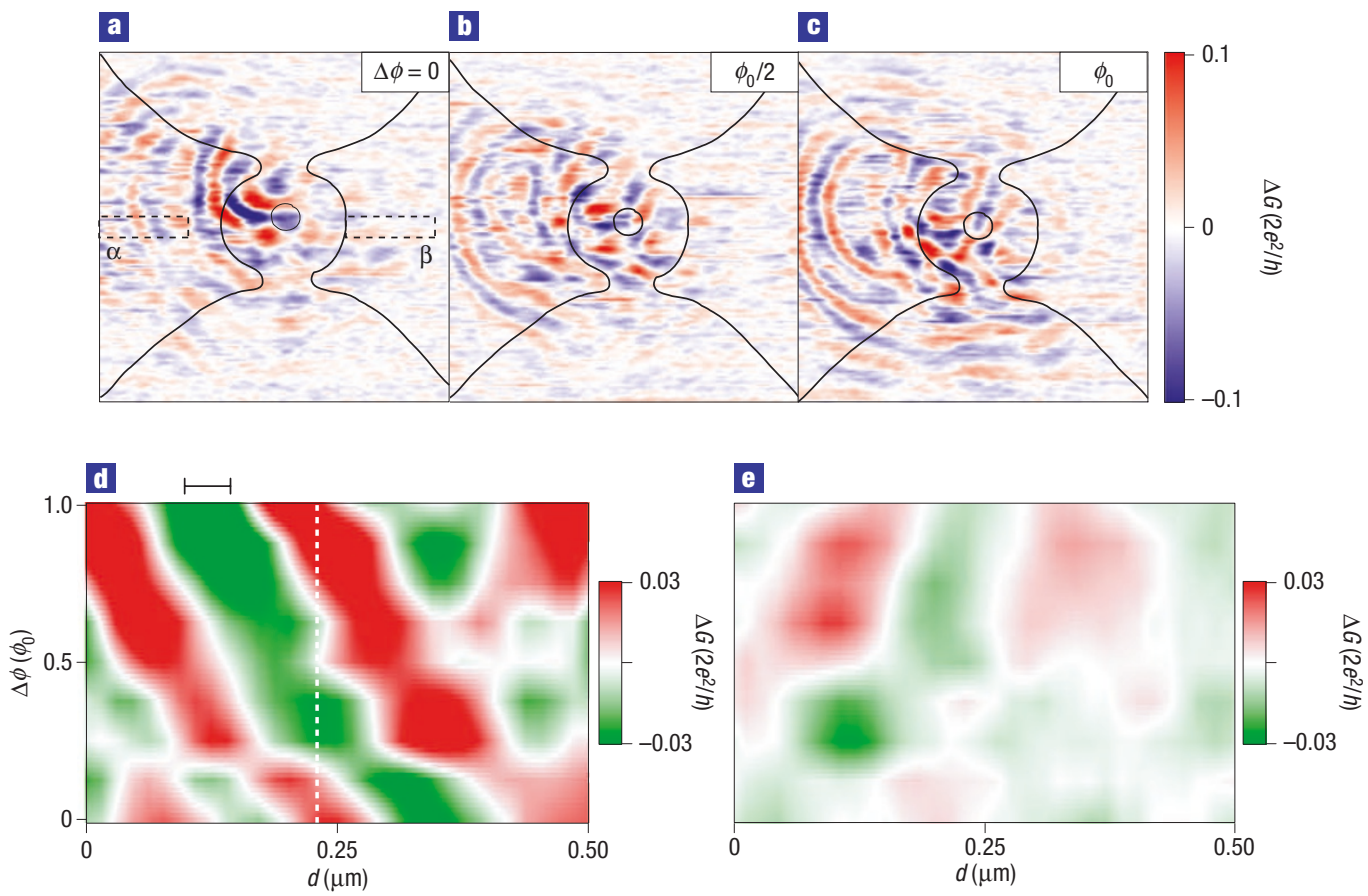

Figure 3 Evolution of conductance maps revealing that concentric fringes originate from the electrostatic $A B$ effect. a-c, Conductance maps recorded at increasing magnetic fields between $B=1.5 \mathrm{~T}$ and $1.526 \mathrm{~T}$ (over one $\mathrm{AB}$ cycle), showing a continuous evolution of fringe patterns. In the vicinity of the ring, the fringe pattern undergoes a cyclic evolution over the sequence. $\mathbf{d}, \mathbf{e}$, Vertical averages of horizontal conductance profiles in regions $\alpha(\mathbf{d})$ and $\beta$ (e) (on both sides of the QR, defined by the dashed rectangles on a), plotted as a function of the phase difference $\Delta \phi$, in units of the AB period $\phi_{0}$. The error bar over $\mathbf{d}$ represents the maximum extent of the lateral movement of the QR over the measurement sequence. The evolutions of the patterns are opposite in the two regions, that is, leftwards in $\mathbf{d}$ and rightwards in $\mathbf{e}$. The cyclic evolution visible in $\mathbf{d}$,e links the concentric fringes to the $A B$ effect, both magnetic and electrostatic. The V-shaped patterns correspond to isophase lines for the electron waves in the $Q R$.

\section{METHODS}

\section{HETEROSTRUCTURE AND 2DES PARAMETERS}

Our InGaAs heterostructure was grown by molecular beam epitaxy. The layer sequence of the heterostructure is as follows: InP substrate, $400 \mathrm{~nm}$ InAlAs buffer, $15 \mathrm{~nm} \mathrm{In}_{0.7} \mathrm{Ga}_{0.3}$ As channel, $10 \mathrm{~nm}$ InAlAs spacer, a $\delta$-doping plane $\left(4 \times 10^{12} \mathrm{Si} \mathrm{cm}^{-2}\right)$ and $15 \mathrm{~nm}$ InAlAs barrier. The 2DES is located at the interface between the channel and spacer layers, that is, $25 \mathrm{~nm}$ below the surface. Measurements on the unpatterned 2DES in the dark yield $100,000 \mathrm{~cm}^{2} \mathrm{~V}^{-1} \mathrm{~s}^{-1}$ for the electron mobility at $4.2 \mathrm{~K}$.

\section{SCANNING GATE MICROSCOPY TECHNIQUE}

The experiments are carried out with a home-made cryogenic AFM, with commercial Pt/Ir-coated Si cantilevers. The movements of the tip in AFM topography mode are detected using a Fabry-Perot optical cavity. The light is switched off in scanning gate microscopy mode, as it affects the electron density and uniformity. After switching the light off, the QR conductance decreases gradually as the electron density decreases. After $2-3 \mathrm{~h}$, it stabilizes, and we can then begin measurements in scanning gate microscopy mode. The tip bias $V_{\text {tip }}$ was adjusted to minimize the variations of the $\mathrm{QR}$ conductance during the scan, so that the system is in the small-perturbation regime. We found that $V_{\text {tip }}=0.3 \mathrm{~V}$ fulfilled this condition. This value is consistent with a surface potential of $\sim 0.3 \mathrm{~V}$ measured by Kelvin probe microscopy on an unpatterned heterostructure region next to the $\mathrm{QR}$.

\section{DATA FILTERING}

We use the following procedure to choose the cut-off frequency $\left(f_{\text {cut }}\right)$ of the filter that we apply to our raw conductance maps. First, as we change $V_{\text {tip }}$, we notice that the background of the conductance map remains essentially unchanged, whereas the position of the small-range features is significantly affected. Therefore, after averaging over a sufficient number of conductance maps acquired with different $V_{\text {tip }}$, the small-range features are averaged away, leaving only the background. We then compare the Fourier transform of the averaged image with a typical Fourier transform of a raw conductance map. We can clearly distinguish between two frequency ranges. The low-frequency range, where the two Fourier transforms are essentially identical, corresponds to the broad background structure in the real-space conductance map. The high-frequency range, where the spectral content is much reduced in the averaged Fourier transform, is related to the small-range features that we want to isolate. The cut-off frequency, $f_{\text {cut }}$, is chosen at the transition between both ranges, that is, $f_{\text {cut }}=4 \mu \mathrm{m}^{-1}$.

\section{Received 6 June 2006; accepted 16 October 2006; published 12 November 2006.}

\section{References}

1. Datta, S. Electronic Transport in Mesoscopic Systems (Cambridge Univ. Press, Cambridge, 1995).

2. Eriksson, M. A. et al. Cryogenic scanning probe characterization of semiconductor nanostructures. Appl. Phys. Lett. 69, 671-673 (1996).

3. Finkelstein, G., Glicofridis, P. I., Ashoori, R. C. \& Shayegan, M. Topographic mapping of the quantum Hall liquid using a few-electron bubble. Science 289, 90-94 (2000).

4. Crook, R., Smith, C. G., Simmons, M. Y. \& Ritchie, D. A. Imaging cyclotron orbits and scattering sites in a high-mobility two-dimensional electron gas. Phys. Rev. B 62, 5174-5178 (2000).

5. Topinka, M. A. et al. Imaging coherent electron flow from a quantum point contact. Science $\mathbf{2 8 9}$, 2323-2326 (2000).

6. Topinka, M. A. et al. Coherent branched flow in a two-dimensional electron gas. Nature 410, 183-186 (2001).

7. Crook, R. et al. Imaging fractal conductance fluctuations and scarred wave functions in a quantum billiard. Phys. Rev. Lett. 91, 246803 (2003).

8. Aharonov, Y. \& Bohm, D. Significance of electromagnetic potentials in the quantum theory. Phys. Rev. 115, 485-491 (1959).

9. Webb, R. A., Washburn, S., Umbach, C. P. \& Laibowitz, R. B. Observation of h/e Aharonov-Bohm oscillations in normal-metal rings. Phys. Rev. Lett. 54, 2696-2699 (1985).

10. Washburn, S., Schmid, H., Kern, D. \& Webb, R. A. Normal-metal Aharonov-Bohm effect in the presence of a transverse electric field. Phys. Rev. Lett. 59, 001791 (1987).

11. Krafft, B., Förster, A., van der Hart, A. \& Schäpers, Th. Control of Aharonov-Bohm oscillations in a AlGaAs/GaAs ring by asymmetric and symmetric gate biasing. Physica E 9, 635-641 (2001). 
12. Hackens, B. et al. Long dephasing time and high-temperature conductance fluctuations in an open InGaAs quantum dot. Phys. Rev. B 66, 241305 (2002).

13. Hackens, B. et al. Dwell-time-limited coherence in open quantum dots. Phys. Rev. Lett. 94, 146802 (2005).

14. Yacoby, A., Sivan, U., Umbach, C. P. \& Hong, J. M. Interference and dephasing by electron-electron interactions on length scales shorter than the elastic mean free path. Phys. Rev. Lett. 66,

1938-1941 (1991).

15. Bozhevolnyi, S. I., Volkov, V. S., Delvaux, E., Laluet, J.-Y. \& Ebbesen, T. W. Channel plasmon subwavelength waveguide components including interferometers and ring resonators. Nature $\mathbf{4 4 0}$ 508-511 (2006).

\section{Acknowledgements}

The authors acknowledge fruitful discussions with M.G. Pala and technical support from J.-F. Motte The experimental setup was built thanks to the initial input of M. Stark. This work was supported by grants from the European Commission (Marie Curie Intra-European Fellowship to B.H.), the
FCT-Portugal (to F.M.) and the CNRS (to V.B.). We acknowledge support from the Action Concertée Nanosciences', French Ministry for Education and Research.

Correspondence and requests for materials should be addressed to B.H. or S.H.

Supplementary Information accompanies this paper on www.nature.com/naturephysics.

\section{Author contributions}

B.H. and F.M. performed the experiments; B.H. fabricated the sample; B.H., F.M., T.O., H.S., J.C., V.B. and S.H. participated in the data analysis; B.H., F.M., T.O., J.C., V.B. and S.H. contributed to the design of the experimental setup; S.B., X.W. and A.C. designed and fabricated the heterostructure substrate; B.H., V.B. and S.H. wrote the article.

\section{Competing financial interests}

The authors declare that they have no competing financial interests.

Reprints and permission information is available online at http://npg.nature.com/reprintsandpermissions/ 